\title{
Análisis factorial de un modelo de socialización y confianza en la dependencia de Internet en estudiantes de secundaria
}

\author{
Factor Analysis of a Model of Socialization and Trust in Internet \\ Dependency among Junior High School
}

\author{
Carlos René Contreras Cázarez (*) renecazarez@gmail.com \\ Gustavo Adolfo León Duarte (*) gustavol@guaymas.uson.mx \\ (*) Universidad de Sonora \\ (Recibido: 31 de julio de 2017; Aceptado para su publicación: 24 de octubre de 2017)
}

Cómo citar: Contreras, C. R. y León, G. A. (2019). Análisis factorial de un modelo de socialización y confianza en la dependencia de Internet en estudiantes de secundaria. Revista Electrónica de Investigación Educativa, 21, e25, 1-13.

doi:10.24320/redie.2019.21.e25.2112

\section{Resumen}

El estudio evalúa la validez y confiabilidad de un modelo estructural restringido en el que confluyen los factores de socialización en línea, confianza y dependencia de Internet en jóvenes de secundaria. La finalidad práctica es explicar la coherencia y pertinencia de los factores que integran el modelo teórico por medio de un análisis de regresiones múltiples y de correlaciones. La muestra la constituyen 4,563 jóvenes de secundarias públicas en México. El análisis factorial reveló que los factores de socialización en línea y confianza afectan directa e indirectamente al factor de dependencia de Internet en los adolescentes, ajustándose de este modo al modelo teórico planteado. Los resultados de las pruebas de las correlaciones mostraron una validez convergente y coeficientes estructurales altos y significativos. Los resultados presentados indican que existe una dependencia de Internet en los jóvenes de secundaria que deviene de sus procesos de sociabilidad, no sólo con sus pares y familiares, sino también con personas desconocidas -como se expone en el modelo probado.

Palabras clave: Comunicación, redes sociales, Internet, dependencia, secundaria.

\section{Abstract}

This study evaluates the validity and reliability of a restricted structural model that brings together factors relating to online socialization, trust, and Internet dependency in junior high students. Its practical purpose is to explain the coherence and relevance of factors in the theoretical model by way of a multiple regression and correlation analysis. The sample was made up of 4,563 students from public junior high schools in Mexico. The factor analysis revealed that factors relating to online socialization and trust have a direct and indirect impact on Internet dependency in adolescents, which is consistent with the theoretical model proposed. The correlation tests showed convergent validity and high and significant structural coefficients. The results indicate that junior high students exhibit Internet dependency resulting from sociability processes not just with their peers and family, but also strangers, as presented in the model tested. 


\section{Introducción}

Internet ha trastocado los escenarios comunes en los que se gestionan, desarrollan y practican las relaciones interpersonales y de comunicación. Los adolescentes que han nacido en entornos tecnológicos y se desenvuelven como verdaderos nativos digitales muestran dos tipos comunes de prácticas sociocomunicativas virtuales, por un lado, la tecnología les facilita el conocimiento sobre el mundo, sustituyendo otras formas tradicionales de aprendizaje y acceso a la información, y por otro lado, Internet y las redes sociales se han convertido para ellos en los escenarios en los que se gestionan todas sus relaciones vitales (de amistad, de ocio, etc.), lo que no está exento de conflictos y riesgos (Hernández y Alcoceba, 2015).

Las redes sociales son percibidas como el elemento integrador indispensable para la construcción de la identidad personal y grupal de los adolescentes (Hernández y Alcoceba, 2015). Sin embargo, otras aportaciones explican que el uso de Internet y las redes sociales son importantes tanto para el desarrollo emocional y social de los jóvenes. Así, el mundo virtual funciona como su patio de recreo para los problemas del desarrollo, tales como la auto-identidad y la sexualidad (Gross, 2004). Es por ello que los estudios de sociabilidad en los jóvenes suscita un creciente interés en investigaciones recientes sobre Tecnologías de la Información y la Comunicación (TIC), como se evidencia en estudios internacionales: Garmendia, Gartaonandia, Martínez y Casado, 2011; Livingstone, 2009; Subrahmanyam, Reich, Waechter y Espinoza, 2008; y en un contexto nacional y local: León et al., 2014, 2015; y León, Contreras y Moreno, 2016; AMIPCI, 2014 e INEGI, 2016. Por otro lado, las redes sociales ofrecen intimidad a los jóvenes, en donde pueden сrecer y madurar en espacios privados (Bartolomé, 2014). En ocasiones se corre el riesgo de que el joven no reciba una orientación adecuada y existe evidencia suficiente que se relaciona negativamente con la pornografía y uso de sustancias (Svedin, Akerman y Priebe, 2011), conducta sexual de riesgo (Carroll et al., 2008; Svedin et al., 2011) y víctimas sexuales (Wolak, Mitchell y Finkelhor, 2007), entre otros peligros.

En clara sintonía con estudios previos realizados por el Grupo de Enseñanza e Investigación en la Comunicación de América Latina (GEIC-AL), concretamente, para el 2014 (León et al., 2014), el presente estudio se propuso profundizar en las variables asociadas a los riesgos de la conectividad en jóvenes de secundaria, su reacción ante los mismos, así como los hábitos o medidas de seguridad que adoptan ellos o sus padres y profesores en la utilización de las TIC. Los informes presentados por el GEIC-AL llegó a la conclusión de que en sus procesos de socialización en línea los jóvenes interactúan a menudo con personas desconocidas como consecuencia de sus procesos de interacción. Sin embargo, se reconoce que otra variable asociada a los riesgos de los adolescentes es la exposición y el acceso al contenido sexual que de manera voluntaria o involuntaria contribuye a la formación sexual de los mismos. Así pues, el estudio de León et al. (2015) informó que 1 de cada 2 menores afirmaron haber recibido imágenes o videos de contenido sexual a través de las redes sociales. Por ello se puede afirmar que las redes sociales no sólo son los medios de comunicación con mayor frecuencia de uso, consumo y utilización por los nativos digitales, sino también son los principales medios de comunicación y difusión personal que son considerados medios privados con un carácter eminentemente íntimo en tanto le permite al adolescente, entre otras cosas, olvidarse de inhibiciones y prohibiciones (León et al., 2015).

Desde una perspectiva internacional, la literatura científica ha evidenciado la presencia del acoso como factor de riesgo para el desarrollo de problemas mentales en los adolescentes. Un estudio presentado por Estévez, Villardón, Calvete, Padilla y Orue (2010) reveló la presencia de al menos una decena de investigaciones que informan que los niños víctimas de acoso manifestaron en algún proceso de su desarrollo un alto grado de tristeza (Camodeca y Goossens, 2005; Hodges y Perry, 1999; Olweus, 1993), así como depresión en el presente (Ivarsson, Broberg, Arvidsson y Gillberg, 2005; Van der Wal, de Wit e Hirasing, 2003) y, finalmente, depresión en su adultez (Olweus, 1991) y baja autoestima (Olweus, 1993; O'Moore y Kirkham, 2001), entre otros problemas relacionados. Los estudios de Internet y la dependencia de esta herramienta, por otra parte, también han suscitado investigaciones en otras latitudes que revelan dicha patología, como el caso de China (Du, Jiang y Vance, 2010; Su, Fang, Miller y Wang, 2011), Corea del Sur (Han et al., 2009); Hong Kong (Shek, Tang y Lo, 2009) y Estados Unidos (Young, 2007). 
En definitiva, la propuesta de un modelo restringido de socialización y confianza en la dependencia de Internet aspira a entender, entre otros aspectos, que Internet puede incrementar los riesgos del menor de edad al estar en línea sin supervisión de un adulto y desarrollar experiencias negativas, como conductas y acciones encaminadas a potencializar el acoso, el hostigamiento y la exposición a la pornografía, entre otras. El objetivo del presente trabajo es evaluar la validez y confiabilidad de un modelo hipotético restringido propuesto para el estudio de socialización y confianza en la dependencia de Internet en jóvenes de secundaria. Así mismo, la finalidad práctica del estudio es explicar la coherencia y pertinencia de los factores del modelo teórico y su relación por medio de indicadores específicos.

\section{Marco teórico}

\subsection{Socialización e interacción}

Diversos autores han definido a la socialización como el proceso en el cual los individuos incorporan normas, roles, valores, actitudes y creencias a partir del contexto socio-histórico en el que se encuentran insertos a través de diversos agentes de socialización, tales como los medios de comunicación, la familia, los grupos de pares y las instituciones educativas, religiosas y recreacionales, entre otros (Arnett, 1995; Maccoby, 2007; Grusec y Hastings, como se cita en Simkin y Becerra, 2013).

En la literatura científica, el concepto de interacción a menudo se encuentra relacionado con las Tic, en especial con Internet. Es posible identificar, en este sentido, que los medios centrales de interacción social en el adolescente son las redes sociales, que con su capacidad de crear comunidades se perfilan como el espacio idóneo no sólo para los estudiantes y sus pares, sino también para la interacción con sus padres, profesores, tutores y personas conocidas y desconocidas. Las relaciones entre padres e hijos suponen aspectos afectivos y cognitivos: apoyo, cariño, disponibilidad para con los hijos. Todos estos elementos tienen que ver con las expectativas, el control, el establecimiento de límites y de orientación de los padres para con sus hijos; en este sentido, estos elementos del contexto familiar contribuyen y son predictivos de adaptación social, desarrollo de competencias sociales y conductas prosociales (Hillaker, Brophy-Herb, Villarruel y Haas, como se cita en Bernal y Rivas, 2011). Por otra parte, es importante mencionar, que es por medio de las interacciones familiares donde se reflejan y transmiten todo tipo de prácticas que se encuentran presentes en un contexto socio cultural.

Los procesos de interacción entre pares conllevan, de acuerdo con Wolf (2008), un conjunto de individuos que comparten categorías sociales comunes y ciertos ámbitos de interacción en tanto grupo. Así, los procesos de socialización e interacción entre grupos de pares se componen principalmente de compañeros de escuela, con los que comparten intereses, grupos deportivos, religiosos y de esparcimiento. En este sentido, los procesos de socialización en línea reconfiguran los marcos para la interacción social, acarreando nuevos retos para la convivencia y la relación de los jóvenes en sus procesos vivenciales. Huertas (2012) explica que los procesos de socialización digital de los adolescentes y jóvenes latinoamericanos son similares a sus espacios de sociabilidad presencial: "Los adolescentes conforman sus relaciones en las redes virtuales con otros jóvenes con los que comparten también espacio físico en el barrio, colegio o instituto" (p. 303). La aparición de las redes virtuales ha reconfigurado los ámbitos de actuación y de relación juveniles, tanto en sus dimensiones productivas (tareas escolares, formación, etc.), como reproductivas (ocio, relaciones familiares y personales) (Hernández y Alcoceba, 2015).

En la región noroeste de México, en Sonora en particular, la situación revela que los servicios de Internet que utilizan los jóvenes de secundaria son variados. Los más utilizados son las redes sociales con un $83 \%$ de preferencias y el uso de mensajes instantáneos (Twitter, WhatsApp, Messenger de Facebook o cualquier aplicación de mensajería multiplataforma que permite enviar y recibir mensajes sin pagar) es lo que prefiere al menos la mitad de los jóvenes que participaron en el estudio (Contreras, León y Moreno, 2014).

\subsection{Comunicación y confianza}

Desde un enfoque comunicativo, la teoría selectiva de Carstensen (1992) sugiere que la experiencia 
emocional es el componente crítico de la interacción social a lo largo de la vida adulta, y su influencia compromete la adaptación de las personas a medida que avanzan en edad. Para Carstensen, las personas (...) seleccionan aquellas interacciones sociales que aportan experiencias emocionales positivas y disminuyen las negativas. De ahí que la interacción social se conciba como una fuente de información que proporciona el alimento emocional necesario para mantener la relación y la propia identidad. Así pues, la confianza es una emoción que se forja en los primeros años de la infancia y, particularmente, en los procesos de socialización primaria. La socialización primaria, por tanto, comporta algo más que un aprendizaje puramente cognoscitivo (Berger, 1997, p. 167). Según los autores de la construcción social de la realidad, sin esa adhesión emocional a otros significantes el proceso de aprendizaje sería difícil, cuando no imposible (Berger y Luckmann, 1968).

Numerosos estudios sobre la familia han señalado aspectos importantes que repercuten en el modo de relacionarse y en la transmisión de valores y emociones. La comunicación familiar, en este sentido, integra el conjunto de los elementos en los procesos de interacción padres e hijos (Maccoby, 2007, p. 34). En las interacciones confluye una serie de aspectos emocionales como el cariño, seguridad, reciprocidad, disponibilidad, comunicación efectiva, confianza, vivencias familiares y rituales que propician o impiden el proceso de socialización y de educación de los menores. Así pues, el tiempo de convivencia coadyuva a la integración de los elementos vivenciales entre padres e hijos, aspectos importantes para el desarrollo emocional y cognitivo de los estudiantes adolescentes.

Del mismo modo, los acuerdos entre padres e hijos son fundamentales para la educación de los menores y para la interacción con ellos. Los acuerdos en este sentido evalúan aspectos afectivos y de control (Chan, Bowes y Wyver, 2009). Por su parte, Rodrigo (2001) se ha referido a la confianza como un contrato pragmático fiduciario, y se refiere a la emoción de la confianza como aquello que se basa en la fe; es decir, en la fe compartida: "Se ha de creer que aquello que se dice es verdad, que ha sucedido realmente asi" (Rodrigo, 1995, pp. 160-163).

\subsection{Dependencia de Internet y apego a dispositivos}

Según Echeburúa y Corral (1994), cualquier conducta normal placentera es susceptible de convertirse en un comportamiento adictivo. Young (1996) ha establecido una serie de criterios para diagnosticar el Síndrome de la Adicción a Internet (IAD, por sus siglas en inglés). Para la autora, la adicción a Internet es un deterioro en el control de su uso que se manifiesta como un conjunto de síntomas cognitivos, conductuales y fisiológicos; es decir, la persona "netdependiente" realiza un uso excesivo de esta herramienta, lo que le genera una distorsión de sus objetivos personales, familiares o profesionales.

En otros estudios, Young (1998), explica que el apego a dispositivos mide y asocia el uso adictivo de Internet por medio de dispositivos móviles como el celular, tableta, etc., que evalúan la compulsión y la dependencia. Así, el indicador de apego a dispositivos permite medir con mayor precisión de una u otra forma problemas relacionados con la ansiedad o irritabilidad en los adolescentes cuando no están conectados a la red por medio del dispositivo celular.

Han sido varias las aportaciones conceptuales que se han adoptado para referirse a este trastorno. La dependencia de Internet se ha conocido con muchos nombres: Desorden de adicción a Internet - (IAD)(Goldberg, 1995), uso compulsivo de Internet (Morahan-Martin y Schumacker, 1997) o Uso patológico de Internet -Pathological Internet Use (PIU)- (Young y Rodgers, 1998). Sin embargo, en otra definición se afirma que la dependencia de Internet se caracteriza por una excesiva o mal controlada preocupación o comportamientos relacionados con el uso de ordenadores y acceso a Internet, que lleva a la alteración de la persona en diferentes niveles (personales, familiares o profesionales) Cao, Su, Liu y Gao (2007).

Otros autores señalan que la dependencia de Internet está asociada al uso y tiempo de conexión. Para Carbonell, Fuster, Chamarro y Oberts (2012), no implica daño psicológico alguno, puesto que no se ha identificado una relación causa-efecto entre el tiempo de uso de Internet y problemas psicológicos. Sin embargo, en su intento por definir la dependencia de Internet, autores como Young (1996) han evidenciado la presencia de algunas consecuencias negativas, como la pérdida o control de los objetivos 
personales, familiares y profesionales, que llevan a un estado de la alteración cuando no se está conectado.

Por su parte, Greenfield (2009) explica que la dependencia a Internet o a las redes sociales está ya instalada cuando hay un apego al dispositivo móvil o un uso excesivo asociado a una pérdida de control. Aparecen síntomas de abstinencia (ansiedad, depresión, irritabilidad) ante la imposibilidad temporal de acceder a la red. Se establece la tolerancia (es decir, la necesidad creciente de aumentar el tiempo de conexión a Internet para sentirse satisfecho) y se producen repercusiones negativas en la vida cotidiana (...) El sujeto muestra ansia por las redes sociales y se produce un flujo de transrealidad (...) (Greenfield, 2009).

\section{Método}

La muestra estuvo integrada por 4,563 estudiantes de secundarias públicas mexicanas del estado de Sonora (México). El universo total de los estudiantes matriculados en las escuelas públicas de la entidad fue de 27,379. La muestra fue seleccionada en función de la representatividad de dicho universo, y teniendo en cuenta variables como distribución del sexo, edad y tipología del centro educativo, entre otras. Para determinar la muestra representativa se utilizaron los siguientes parámetros: error máximo aceptable (2\%); porcentaje estimado de la muestra (99\%); nivel deseado de confianza (99\%); tamaño del universo (27,379 estudiantes).

Como se ha evidenciado en anteriores publicaciones (León et al., 2016), el instrumento forma parte de un estudio macro de Internet y relaciones interactivas en jóvenes de secundarias públicas mexicanas, el cual mide las variables de acceso, hábitos, competencias, sociabilidad, confianza, riesgos y dependencia de Internet. Para efecto de la validez y confiabilidad del modelo estructural restringido propuesto, se tomó el factor de socialización en línea, el cual está constituido por 24 ítems que señalan los procesos de interacción entre pares -compañeros de clase, familiares y personas desconocidas.

Otro de los factores que conforman al modelo restringido es la confianza, 29 ítems que miden los acuerdos pragmáticos entre padres e hijos, así como la comunicación y tiempo de convivencia de calidad entre los mismos. Por último, el factor dependencia de Internet está conformado por 21 ítems que miden el apego a los dispositivos tecnológicos móviles -particularmente la telefonía celular, la preocupación constante de no estar conectado y, finalmente, el tiempo de uso. En cualquiera de los casos, para responder a los ítems el encuestado debía señalar cada una de las afirmaciones mediante una escala tipo Likert con cinco alternativas de respuesta: ( $0=$ nunca, $1=$ rara vez, $2=$ =algunas veces, $3=$ =casi siempre y $4=$ siempre.)

\subsection{Procedimiento y análisis}

Los datos del estudio se sometieron a un Análisis Factorial Exploratorio (AFE) en el programa estadístico SPSS versión 21 para conocer el número de factores que subyacen a las relaciones entre los factores, así como determinar las variables propuestas que conforman al modelo. Fue necesario hacer un AfE para la validación de los constructos y la consistencia interna del mismo. Se realizó primero un análisis de factores principales con el método de rotación Varimax en el conjunto completo de ítems, estableciendo las parcelas de todos los factores que componen al instrumento. De esta forma, los valores y las parcelas se utilizaron para determinar el número de factores a extraer en el modelo restringido para el estudio en cuestión. Dado el tamaño de la muestra y el alfa de las escalas se realizó a la extracción de los factores (tabla I). 
Tabla I. Análisis de confiabilidad de la escala

\begin{tabular}{l|c}
\hline Escala & Alfa \\
\hline Interacción con los pares & .92 \\
Interacción con desconocidos & .77 \\
Interacción con los padres & .89 \\
Apego al dispositivo & .71 \\
Acuerdos pragmáticos & .91 \\
\hline Tiempo de uso & .81 \\
Preocupación constante & .89 \\
\hline Tiempo de convivencia & .78 \\
Comunicación familiar & .63 \\
\hline
\end{tabular}

Рara el Análisis Factorial Confirmatorio (AFC) se utilizó el programa EQS versión 6.1, donde se analizó la coherencia y validación de los factores determinando la relación y direccionalidad de los mismos. Los índices de ajuste adoptados fueron el índice de Ajuste Comparativo (CFI) (Bentler, 1990), el índice de Bentler-Bonett de Ajuste No Normado (NNFI) (Bentler y Bonett, 1980) y la Raíz Cuadrada del Error Medio Cuadrático (RMSEA) (Steiger, 1990).

En la literatura estadística, un CFI aceptable debe ser >.90 (Bentler, 1992), para el análisis en cuestión los valores fueron mayores a este respecto en contraste al modelo inclusivo (ver tabla II.) Sin embargo, la literatura estadística ha establecido que estos índices de ajuste no son inmunes a la falta de especificación de un modelo (Heene, Hilbert, Draxler, Ziegler y Bühner, 2011; Marsh, Hau y Wen, 2004), por ello en este estudio los criterios para los índices de ajuste del modelo propuesto restringido de tres factores fueron tratados como guías y no como valores. Las consistencias internas de los tres factores propuestos para el modelo estructural restringido se calcularon utilizando coeficientes estructurales y alfa de Cronbach.

Tabla II. Comparación de los Índices de Bondad de Ajuste del modelo inclusivo y restringido

\begin{tabular}{l|c|c|c|c|c|c|c|c}
\hline \multicolumn{1}{c|}{ Modelos } & $\mathrm{X}^{2}$ & $\mathrm{GL}$ & $\mathrm{P}$ & $\mathrm{BBNFI}$ & $\mathrm{BBNNFI}$ & CFI & RMSEA & $\mathrm{R}^{2}$ \\
\hline $\begin{array}{l}\text { Modelo inclusivo (Acceso y } \\
\text { uso, socialización y } \\
\begin{array}{l}\text { confianza para explicar la } \\
\text { Dependencia de Internet) }\end{array}\end{array}$ & 54.99 & 24 & 00 & .86 & .87 & .91 & .08 & .88 \\
\hline $\begin{array}{l}\text { Modelo Restringido } \\
\text { (Socialización en línea y } \\
\text { confianza en la } \\
\text { dependencia de Internet.) }\end{array}$ & 25.99 & 24 & .14 & .92 & .95 & .91 & .08 & .68 \\
\hline
\end{tabular}

Nota: Elaborado por Contreras (2017), adaptado del modelo de León et al. (2016). 


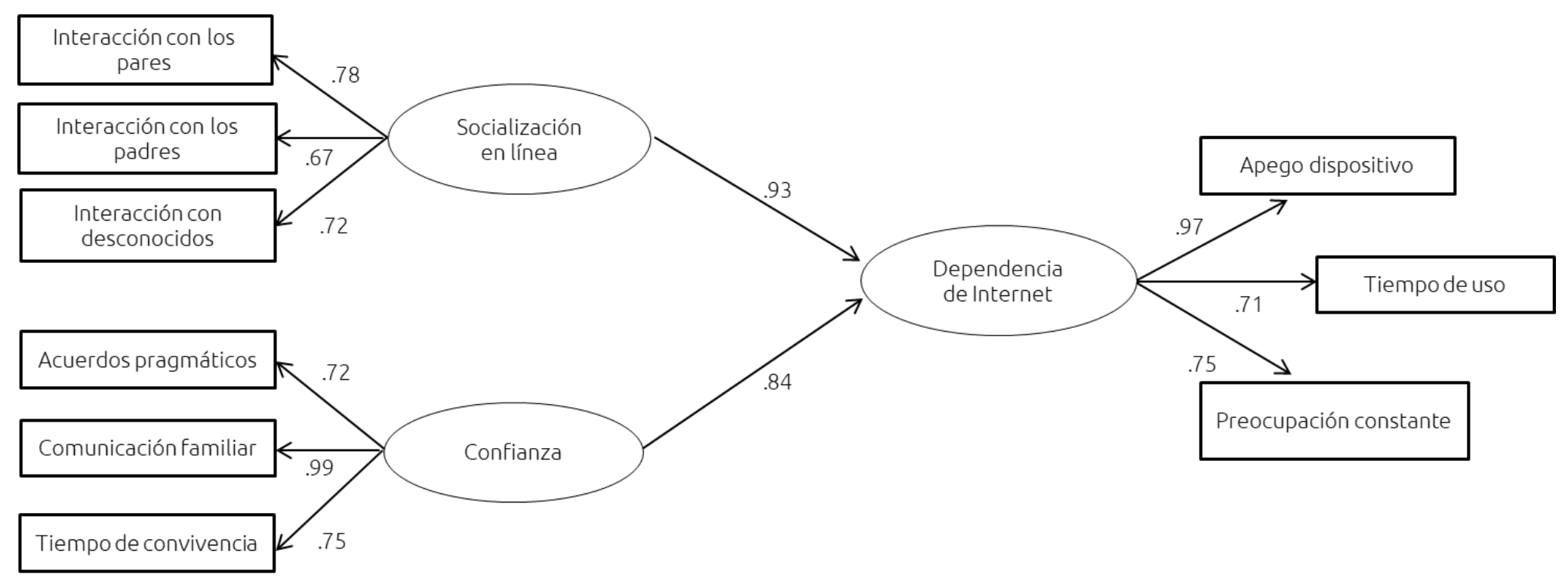

Elaborado por Contreras (2017)

$\mathrm{X}^{2}=25.99 ; \mathrm{GL}=24 ; \mathrm{P}=.14 ; \mathrm{BBNFI}:=.92 ; \mathrm{BBNNFI}:=.95 \mathrm{CFI}:=.91 ; \mathrm{RMSEA}=.08 ; \mathrm{R}^{2} .68$

Figura 1. Modelo restringido de socialización en línea y confianza para explicar la dependencia de Internet en jóvenes en México 


\section{Resultados}

El modelo estructural muestra que los factores del modelo restringido de socialización en línea y confianza propuestos para este estudio de dependencia de Internet se formaron congruentemente con sus indicadores, lo que muestra la validez del constructo de las variables latentes del estudio. De igual forma, los valores altos y significativos entre los indicadores del modelo y los factores propuestos revelan validez convergente.

Los coeficientes alfa para todas las escalas del modelo fueron mayores a .60 (ver tabla I), por lo que se asume que las mismas presentan un considerable grado de confiabilidad. La figura 1 muestra la relación del modelo estructural restringido, las variables latentes se representan mediante círculos y las variables observadas en rectángulos, mientras que la relación entre cada uno de los factores del modelo se representa con flechas, con un alto coeficiente estructural de afectación a la variable dependiente (VD).

El factor de socialización en línea se formó con la variable Interacción con los pares (con un peso factorial de .78), la variable Interacción con los padres (.67) e Interacción con personas desconocidas (.72); y el factor de confianza con las variables de Acuerdos pragmáticos (.72), Comunicación familiar (.99) y Tiempo de convivencia (.75); por último, el factor de dependencia de Internet estuvo conformado por las variables de Apego al dispositivo (.97), Tiempo de uso (.71) y Preocupación constante (.75)

Como puede apreciarse en el modelo, el factor de socialización en línea (conformado por las variables Interacción con pares, Interacción con padres e Interacción con desconocidos) tuvo un efecto fuerte y significativo de coeficiente estructural (.93) en la vD dependencia de Internet; mientras que el factor de confianza (por las variables conformadas por Acuerdos pragmáticos, Comunicación familiar y Tiempo de convivencia) mostró un coeficiente estructural de (.84) en la VD.

De este modo, todos los indicadores de bondad de ajuste del modelo hipotético restringido alcanzaron los valores requeridos para la congruencia y pertinencia del modelo. La $x^{2}$ fue baja y no significativa al mostrar una ( $p=.14)$, los índices prácticos NNFI, así como el CFI fueron mayores a .90, como se muestra en el modelo. RMSEA tuvo un valor de .08 y la $\mathrm{R}^{2}$ del modelo fue de .68 , lo que explica el $68 \%$ de la varianza del modelo.

\section{Discusión y conclusiones}

De acuerdo a los resultados que arrojó el modelo estructural restringido de socialización y confianza en la dependencia de Internet, los jóvenes de secundaria público incluidos en la muestra presentan un efecto fuerte y significativo en la dependencia de Internet. En consonancia, el factor de confianza presentó un efecto directo positivo con la VD. Todo apunta a que mediante los acuerdos familiares, así como la comunicación familiar eficiente y tiempo de convivencia en la misma, contribuiría de manera razonable para inhibir o disminuir la dependencia de esta herramienta en los jóvenes. Por consiguiente, se debe tener en cuenta que es en el proceso de socialización por medio de las redes sociales donde el adolescente -si bien construye su identidad y su sentido de pertenencia-, es también el entorno donde mayoritariamente se expone a los posibles riesgos, particularmente cuando interactúa o entra en contacto con personas desconocidas. Existen, por otra parte, otros factores de riesgos que se han señalado en los procesos de socialización en línea de los menores. Por ejemplo, el acoso y el hostigamiento, así como la exposición de contenido sexual inapropiado para un menor de edad. Así pues, algunos usos desfavorables de Internet se relacionan con el proceder de los mismos jóvenes -cuando están solos sin supervisión de un adulto.

Sin embargo, la teoría establece que la dependencia de Internet en los adolescentes es un fenómeno que va en aumento y deviene, como se ha argumentado en otros estudios, por el acceso y equipamiento en el hogar, particularmente de los dispositivos móviles (León et al., 2016). Se establece también que la prevalencia del uso excesivo de esta herramienta en la región proviene de los procesos de sociabilidad, particularmente en la población adolescente (Contreras, León y Moreno, 2014). Del mismo modo, es comprensible que el factor de confianza haya resultado con un efecto directo positivo en la VD, si se 
considera que los lazos afectivos pueden contribuir de manera significativa al desapego de los dispositivos móviles y la disminución del tiempo de uso de Internet, al llegar a un acuerdo entre padres e hijos, o bien, compartir tiempo de calidad como parte de la convivencia familiar y la comunicación.

El modelo restringido explicó el 68\% de la varianza de la dependencia de Internet. Es decir, que cerca de una tercera parte de la socialización en línea de los jóvenes de secundaria se lleva a cabo por medio de sus interacciones en línea con sus iguales, familiares y desconocidos, afectando directamente a la VD, incrementando su tiempo de uso, el apego a los dispositivos y una preocupación constante cuando no se está conectado. Esto explicaría por qué la mayoría de los adolescentes prefiere socializar en redes sociales, como Facebook, WhatsApp y otras, según el estudio presentado por León et al. $(2014,2015)$; en el mismo informe se observó que las redes sociales tenían mayor afluencia por parte de los menores, como parte de su uso, consumo y utilización. De este modo, queda claro que las principales actividades de los estudiantes en Internet son las interacciones sociales en línea. Así pues, Internet refuerza los lazos amistosos con personas con las que se tiene un contacto físico, frecuente y previo, como son sus compañeros de clase y la familia, pero también con personas desconocidas.

El estudio se interesó de manera particular en el factor confianza, por ser una emoción que se construye principalmente en lazos afectivos. Sin embargo, pese que su relación con la variable de dependencia de Internet fue directa y positiva con un coeficiente estructural de .84 , se asume que de existir una buena comunicación y tiempo de convivencia de calidad entre los responsables de la educación del menor y los mismos jóvenes, se esperaría una disminución en el apego a los dispositivos móviles y el tiempo de uso de Internet por parte de los adolescentes. El estudio sobre jóvenes y medios digitales móviles en México (León et al., 2015) reveló que los estudiantes tienen poca o nula comunicación con sus padres, y pese a que sienten que el tiempo de convivencia que pasan con la familia en ocasiones es suficiente, la comunicación no lo es tanto, afirmaron los adolescentes en el estudio. En la misma línea, el informe permitió concluir, en relación con la confianza que los adolescentes de secundarias públicas en Sonora tienen, que hay mayor confianza con los amigos que con sus padres. No obstante, es con los compañeros de clase con los que mantienen mayor comunicación, tanto en redes sociales como de manera presencial en la escuela.

A fin de aumentar el poder explicativo del modelo restringido propuesto y propiamente dicho del modelo inclusivo (León et al., 2016), es recomendable que en futuros estudios se incluyan variables e indicadores que la literatura considera como relacionadas o asociadas con la dependencia de Internet en los adolescentes. Tomando en consideración que la validez y confiabilidad de los factores propuestos en el modelo restringido aquí presentado resultó con indicadores aceptables, consideramos que las escalas expuestas deben proporcionar una buena base para la investigación futura. Una de las limitaciones que presenta el estudio aquí presentado es que la muestra la constituyen únicamente estudiantes de secundarias públicas mexicanas, por lo que se sugiere extender la muestra a otros sectores de la población juvenil para contrastar o, en su defecto, confirmar los resultados y lograr una generalización de los datos.

\section{Referencias}

Arnett, J. J. (1995). Broad and narrow socialization: The family in the context of a cultural theory. Journal of Marriage and the Family, 57(3), 617-628

Asociación Mexicana de la Industria Publicitaria y Comercial en Internet. (2014). Estudio sobre los hábitos de los usuarios de Internet en México. Recuperado de https://www.asociaciondeinternet.mx/es/noticiasx/2160-estudio-amipci-de-habitos-de-los-usuarios-deinternet-en-mexico-2014

Bartolomé, J. (2014). Redes sociales y cuidado de la intimidad en adolescentes y familias: una propuesta educativa. Universidad de Murcia. 
Bentler, P. M. (1990). Comparative fit indexes in structural models. Psychological Bulletin, 107(2), 238-246. Recuperado de https://psycnet.apa.org/record/1990-13755-001

Bentler, P. M. (1992). On the fit of models to covariances and methodology to the Bulletin. Psychological Bulletin, 112(3), 400-404. Recuperado de https://psycnet.apa.org/record/1993-12081-001

Bentler, P. M. y Bonett, D. G. (1980). Significance tests and goodness of fit in the analysis of covariance structures. Psychological Bulletin, 88(3), 588-606. Recuperado de https://psycnet.apa.org/record/1981$\underline{06898-001}$

Berger, T. (1997). Observation and analysis of small-scale solar magnetic structure. Tesis de doctorado, Stanford University.

Berger, P. y Luckmann, T. (1968). La construcción social de la realidad. Madrid: Amorrortu.

Bernal, A. y Rivas, S. (2011). Relaciones padres e hijos. Un análisis de la realidad española a partir de la Encuesta "La familia, recurso de la sociedad". Recuperado de https://dadun.unav.edu/bitstream/10171/23646/1/Bernal\%20y\%20Rivas RELACl\%C3\%93N\%20PADRES $\% 20 \mathrm{E} \% 20 \mathrm{HIJOS.pdF}$

Camodeca, M. y Goossens, F. A. (2005). Aggression, social cognitions, anger and sadness in bullies and victims. Journal of Child Psychology and Psychiatry, 46(2), 186-197.

Cao, F., Su, L., Liu, T. y Gao, X. (2007). The relationship between impulsivity and Internet addiction in a sample of chinese adolescents. European Psychiatry, 22(7), 466-471.

doi:10.1016/i.eurpsy.2007.05.004

Carbonell, X., Fúster, H., Chamarro, A. y Oberst, U. (2012). Adicción a internet y móvil: una revisión de estudios empíricos españoles. Papeles del Psicólogo, 33(2), 82-89. Recuperado de

http://www.papelesdelpsicologo.es/pdf/2096.pdf

Carroll, J. S., Padilla-Walker, L. M., Nelson, L. J., Olson, Ch. D., Barry, C. M. y Madsen, S. D. (2008). Generation XXX, pornography acceptance and use among emerging adults. Journal of Adolescent Research, 23(1), 6-30. https://doi.org/10.1177/0743558407306348

Carstensen, L. L. (1992). Social and emotional patterns in adulthood: support for socioemotional selectivity theory. Psycology and Aging, 7(3), 331-338. Recuperado de https://psycnet.apa.org/buy/1993$\underline{01043-001}$

Chan, S. M., Bowes, J. y Wyver, S. (2009). Parenting style as a context for emotion socialization. Early Education and Development, 20(4), 631-656. doi:10.1080/10409280802541973

Contreras, C. R., León, G. A. y Moreno, D. E. (2014). Internet en jóvenes de secundaria. Un estudio de sociabilidad en las relaciones interactivas en Hermosillo, Sonora, México. Revista Internacional de Tecnologías en la Educación, 1(1), 57-66. Recuperado de https://journals.epistemopolis.org/index.php/tecnologiasedu/article/view/1099

Du, Y. S., Jiang. W. y Vance, A. (2010). Longer term effect of randomized, controlled group cognitive behavioral therapy for Internet addiction in adolescent students in Shanghai. Australian and New Zealand Journal of Psychiatry, 44,129-134.

Echeburúa, E. y Corral, P. (1994). Adicciones psicológicas: más allá de la metáfora. Clínica y Salud, 5(3), 251-258. Recuperado de https://journals.copmadrid.org/clysa/art/07e1cd7dca89a1678042477183b7ac3f 
Estévez, A., Villardón, L., Calvete, E., Padilla, P. y Orue, I. (2010, enero). Adolescentes víctimas de cyberbullying: prevalencia y características. Behavioral Psychology/Psicología Conductual, 1(18), 73-89.

Garmendia, M., Gartaonandia, C. Martínez, G. y Casado, M. A. (2011). Riesgos y seguridad en Internet: los menores españoles en el contexto europeo. Universidad del País Vasco.

Goldberg, I. (1995). Internet addiction disorder. Diagnostic criteria. Recuperado de http://www.iucf.indiana.edu/brown/hyplan/addict.html

Gross, E. (2004). Adolescent Internet use. What we expected, what we report? Applied Developmental Psychology, 25, 633-649.

Han, D. H., Lee, Y. S., Na, C., Ahn, J. Y., Chung, U. S., Daniels, M. A. et al. (2009).The effects of methylphenidate on Internet video game play in children with attentiondeficit/hyperactivity disorder. Comprehensive Psychiatry, 50(3), 251-256. Recuperado de https://www.ncbi.nlm.nih.gov/pubmed/19374970

Heene, M., Hilbert, S., Draxler, C., Ziegler, M. y Bühner, M. (2011). Masking misfit in confirmatory factor analysis by increasing unique variances: a cautionary note on the usefulness of cutoff values of fit indices. Psychological Methods, 16, 319-336.

Hernández, C. y Alcoceba, J. A. (2015). Socialización virtual, multiculturalidad y riesgos de los adolescentes latinoamericanos en España, Icono, 14(13), 116-141. doi:10.7195/ri14.v13i2.787

Hodges, E. V. y Perry, D. G. (1999). Personal and interpersonal antecedents and consequences of victimization by peers. Journal of Personality and Social Psychology, 76, 677-685.

Huertas, A. (2012). Procesos de sociabilidad e identidades en Internet: una aproximación a partir del estudio de contextos sociales multiculturales juveniles en España. D. Cogo, M. ElHajji y A. Huertas (Coords.). Diásporas, migrações, tecnologias da comunicação e identidades transnacionais [Diásporas, migraciones, TIC e identidades transnacionales]. Barcelona: InComUAB.

INEGI. (2016). Encuesta nacional sobre disponibilidad y uso de tecnologías de la información en los hogares 2015. Recuperado de

http://www.inegi.org.mx/saladeprensa/boletines/2016/especiales/especiales2016 03 01.pdf

Ivarsson, T., Broberg, A. G., Arvidsson, T. y Gillberg, C. (2005). Bullying in adolescence: psychiatric problems in victims and bullies as measured by the Youth Self Report (YSR) and the Depression Self-Rating Scale (DSRS). Nordic Journal of Psychiatry, 59, 365-373.

Kaiser, H. F. (1958). The varimax criterion for analytic rotation in factor analysis. Psychometrika, 23(3), 187201.

León, G. y Contreras, C. R. (2014). Tecnologías de la Comunicación y la Información, educación y jóvenes de secundaria en México. Un estudio sobre socialización y construcción de la identidad de las relaciones interactivas en jóvenes de secundaria. En E. Soriano, A. J. González y Cala, V. C. (Eds.), Retos actuales de Educación y Salud Transcultural (tomo I). Universidad de Almería.

León, G., Caudillo, Y., Contreras, R. y Moreno, D. (2014). Internet seguro y jóvenes de secundaria en México. Universidad de Sonora-SEP.

León, G., Caudillo, D. Y., Contreras, C. R. y Moreno, D. E. (2015). Jóvenes y medios digitales móviles en México. Un estudio de variables asociadas en perspectiva Interdisciplinar (1a. ed.). México: Pearson. 
León, G., Contreras, C. R. y Moreno, D. (2016). Probando modelos interdisciplinares inclusivos en la dependencia de Internet en Jóvenes. Nuevas variables asociadas. Revista Latina de Comunicación Social, 71, 616-631. doi:10.4185/RLCS-2016-1112

Livingstone, S. M. (2009). Children and the Internet: great expectations, challenging realities. Cambridge, MA: Polity.

Maccoby, E. E. (2007). Historical overview of socialization research and theory, en J. E. Grusec y P. D. Hastings (Eds.), Handbook of socialization: theory and research (pp. 13-41). Nueva York: Guilford Press.

Marsh, H. W., Hau, K. T. y Wen, Z. (2004). In search of golden rules: Comment on hypothesis-testing approaches to setting cutoff values for fit indexes and dangers in overgeneralizing Hu and Bentler's (1999) findings. Structural Equation Modeling: A Multidisciplinary Journal, 11, 320-341.

Morahan-Martin, J. M. y Schumacker, P. (1997). Incidence and correlates of pathological internet use. Documento presentado en la 105th Anual Convention of de la American Psychological Association, Chicago, IL.

Olweus, D. (1991). Bully/victim problems among schoolchildren: Basic facts and effects of a school-based intervention program. En K. Rubin y D. Pepler (Dirs.), The Development and Treatment of Childhood Aggression (pp. 411-448). Hillsdale, NJ: Erlbaum.

Olweus, D. (1993). Victimization by peers: Antecedents and long-term outcomes. En K. H. Rubin y J. B. Asendorpf (Dirs.), Social withdrawal, inhibition, and shyness in childhood (pp. 315-341). Hillsdale, NJ: Erlbaum.

O'Moore, M. y Kirkham, C. (2001). Self-esteem and its relationship to bullying behaviour. Aggressive Behavior, 27, 269-283.

Rodrigo, M., (1995). Los modelos de la comunicación. Madrid: Tecnos.

Rodrigo, M. (2001). Teorías de la comunicación. Ámbitos, métodos y perspectivas. Barcelona: Aldea global.

Shek, D. T., Tang, V. M. y Lo, C. Y. (2009). Evaluation of an Internet addiction treatment program for Chinese adolescents in Hong Kong. Adolescence, 44, 359-373.

Simkin, H. y Becerra, G. (2013) El proceso de socialización. Apuntes para su exploración en el campo psicosocial Ciencia, Docencia y Tecnología, XXIV(47), 119-142. Recuperado de

http://www.redalyc.org/pdf/145/14529884005.pdf

Steiger, J. H. (1990). Structural model evaluation and modification: an interval estimation approach. Multivariate Behavioral Research, 25, 173-180.

Subrahmanyam, K., Reich, S. M., Waechter, N. y Espinoza, G. (2008). Online and offline social networks: use of social networking sites by emerging adults. Journal of Applied Developmental Psychology, 29(6), 420-433.

Svedin, C., Akkerman, I. y Priebe, G. (2011). Frequent users of pornography. A population based epidemiological study of Swedish male adolescents. Journal of adolescence, 34(4), 779-788.

Su, W., Fang, X., Miller J. K. y Wang, Y. (2011). Internet-based intervention for the treatment of online addiction for college students in China: a pilot study of the Healthy Online Self-helping Center. Cyberpsychology Behavior Social Network, 14(9), 497-503. 
Van der Wal, M. F., de Wit, C. A. M. y Hirashing, R. A. (2003). Psychosocial health among young victims and offenders of direct and indirect bullying. Pediatrics, 111, 1312-1317.

Wolak, J., Mitchell, K. J. y Finkelhor, D. (2007). Does online harassment constitute bullying? An exploration of online harassment by known peers and online-only contacts. Journal of Adolescent Health, 41(6), 51-58.

Wolf, S. (2008). Peer groups: expanding our study of small group communication. Thousand Oaks, CA: Sage.

Young, K. S. (1996, agosto). Internet addiction: the emergence of a new clinical disorder. Trabajo presentado en la 104th annual meeting of the American Psychological Association. Toronto, Canadá.

Young, K. S. (1998). Caught in the net: how to recognize the signs of Internet addiction-and a winning strategy for recovery. Nueva York: Wiley.

Young, K. S. y Rodgers, R. C. (1998). The relationships between depression and Internet addiction. Cyberpsychology and Behavior, 1, 25-28.

Young K. S. (2007). Cognitive behavior therapy with Internet addicts: treatment outcomes and implications. Cyberpsychology and Behavior, 10, 671-679. 